\title{
Can Major Depression Improve the Perception of Visual Motion?
}

\author{
Pascal Wallisch and Romesh D. Kumbhani \\ Center for Neural Science, New York University, New York, New York 10003 \\ Review of Golomb et al.
}

It has always been the promise of basic research to eventually bring its dearly earned knowledge to bear on practical matters. At the same time, it is understood that one should first get a good grasp of the complex workings of the healthy nervous system before being able to meaningfully treat its disorders.

Unfortunately, the need to alleviate human suffering resulting from neural disorders is a pressing concern. An estimated 14,000,000 American adults experience at least one episode of major depressive disorder (MDD) in a given year (Kessler et al., 2003). People suffering from MDD typically exhibit a complex pattern of symptoms, which can render the assessment of patients difficult. Additionally, treatments for this disorder are often of limited efficacy or are outright contentious. Thus, major depression poses an urgent and formidable challenge for mental health practitioners as well as the larger neuroscience community. At the same time, the neural basis of MDD remains largely unknown or controversial.

In contrast, basic research into visual perception, motion perception in particular, has made astonishing strides in past decades. Thus, the neural system respon-

Received Sept. 14, 2009; revised 0ct. 12, 2009; accepted 0ct. 14, 2009. We thank R. Dotson and S. H. Sokol for helpful discussion when preparing this manuscript.

Correspondence should be addressed to Pascal Wallisch, Center for Neural Science, New York University, 4 Washington Place, Room 809, New York, NY 10003. E-mail: pascal.wallisch@nyu.edu.

DOI:10.1523/JNEUROSCI.4560-09.2009

Copyright $\odot 2009$ Society for Neuroscience $\quad$ 0270-6474/09/2914381-02\$15.00/0 sible for the perception of visual motion is extremely well understood in terms of its basic anatomy, physiology, and underlying computational principles (Born and Bradley, 2005). In addition, visual motion perception has also been extensively studied psychophysically.

In a recent paper, Golomb et al. (2009) attempted to link these traditionally rather separated fields. The crux of their work is based on previous psychophysical research by Tadin et al. (2003) that uncovered a peculiar effect of contrast and size on performance in a visual motion task. Briefly, Tadin et al. (2003) presented visual stimuli (drifting gratings) to human observers and asked them to indicate the direction of motion. They systematically varied contrast and size of the stimuli and measured the minimal presentation time necessary to reliably distinguish the direction of motion (duration threshold). Interestingly, they found that a large, high-contrast stimulus required longer presentation times for its direction to be discerned than was required for either a small, high-contrast stimulus or a large, lowcontrast stimulus.

This surprising finding, known as spatial suppression, has been linked to the surround suppression of area MT neurons, which presumably results from the action of local GABA-mediated inhibitory connections (Betts et al., 2005). Further, it has been shown via nuclear magnetic resonance spectroscopy that depressed patients exhibit a significant decrease of GABA levels in their occipital cortices relative to controls (Sanacora et al., 1999).
Golomb et al. (2009) built on this preexisting knowledge by using MDD as a natural experimental intervention. They wondered whether the decreased GABA levels in people previously suffering from MDD could have behavioral implications. Specifically, they hypothesized that these patients might have reduced spatial suppression due to weakened local inhibitory connections in area MT.

To address this question, they asked subjects to judge the direction of slowly moving sinusoidal gratings and computed psychophysical thresholds as performance metrics, comparing subjects with at least two past MDD episodes to control subjects without a history of depression. They found that while both groups exhibited spatial suppression, the effect was substantially reduced in previously depressed subjects. In addition, the authors established a "dose-dependent" relationship—subjects who reported longer periods of depression exhibited less spatial suppression. These results suggest that lower GABA levels in depressed subjects have functional implications for the perception of visual motion in a way that is consistent with reduced spatial suppression. Since reduced GABA levels remain low long after recovery from depression (Bhagwagar et al., 2007), the authors propose that this measure could be used as a stable indicator of vulnerability to depression.

It is important to emphasize that this is not the first time that a link between basic motion vision and mental or neurological disorders has been attempted (Spencer et al., 2000; Tadin et al., 2006). However, clin- 
ical studies of this kind come with their own set of challenges. In particular, baseline performance is often different between experimental and control groups because of the cognitive strain from the mental illness. Having unequal baselines typically compromises the experimental logic, making further comparisons hard to interpret. Golomb et al. (2009) elegantly avoided this problem, as their subjects had recovered from MDD but still had reduced GABA levels. Thus, experimental and control groups exhibited comparable baseline levels on a variety of psychological and psychophysical measures, including intelligence quotient, basic visual function, and contrast sensitivity.

While Golomb et al. (2009) should be commended for their valiant attempt, we have several concerns regarding the interpretation of their results. Our enthusiasm was somewhat dampened by the rather indirect experimental logic. From the standpoint of basic experimental design, the study would have been more compelling if the authors had measured individual GABA levels as well as individual measures of depression to see whether there is a covariation between GABA levels, severity of depression, and spatial suppression. Strictly speaking, it is unclear whether the effect reported by the authors is caused by changes in GABA. In the original study by Sanacora et al. (1999), GABA concentrations were only measured within a $3 \times 3 \times 1.5 \mathrm{~cm}$ area over the midline of the occipital lobe, presumably over foveal primary visual cortex (area V1). Although a reduced inhibitory drive in V1 could affect downstream visual processing, the neural mechanism by which it might selectively reduce surround suppression in area MT has not been conclusively established, nor is there direct evidence that local inhibition in area MT is actually impaired. Additionally, it is quite conceivable that another consequence of depression could account for the differences in spatial suppression, regardless of GABA levels in area V1. Recent work by Anton-Erxleben et al. (2009) has shown that attention directed away from the receptive field of an MT neuron can reduce its surround suppression. Furthermore, it is unclear whether GABA levels decrease with the duration of the depressive episode, even though the magnitude of spatial suppression was found to covary with depression duration. Finally, it has been pointed out that spatial suppression might not be linked to the strength of the MT surround at all, rather that it can entirely be explained by the relative contrast of the stimulus to contrast thresholds at different sizes (Aaen-Stockdale et al., 2009). Although it is conceivable that contrast thresholds may change as a result of depression, currently there is no evidence bearing on this matter.

Even so, the study by Golomb et al. (2009) provides an important bridgehead to further integrate the study of mental illness and visual psychophysics. Currently the link between the two domains remains virtually unexplored. It would be useful to show how the low levels of GABA in patients with MDD manifest themselves in other well established visual tasks. For example, GABAmediated local inhibition is thought to sharpen the direction tuning of MT neurons (Thiele et al., 2004). One would predict broader direction tuning curves and implications for performance in a fine direction discrimination task if this inhibition were weakened. As the authors only did a coarse discrimination task (subjects distinguished vertical gratings moving either left or right), if this predicted effect bears out, it would be a powerful corroboration of their results. If this link were confirmed, the use of recovered depressed subjects would likely become much more common in visual psychophysics. Many of the models built from psychophysical data rely on stages of local inhibition. However, the psychophysical data in itself is often insufficient to constrain or rule out different versions of these models. Using subjects with lower cortical GABA levels could provide an elegant way to test these models.

Because of the pioneering nature of Golomb et al. (2009) in tying research on nuclear magnetic resonance spectroscopy, psychophysics, and major depression together, many questions naturally remain open. This allows one to speculate about exciting questions. For instance, it is unclear whether GABA levels in depressed individuals are lowered only in occipital cortex or perhaps also in temporal and parietal lobes. Maybe low GABA levels predispose individuals to mental illness as well as other manifestations of low lateral inhibitions. Notably, a link between heightened creativity and higher probability of developing mental depression has been suggested for many years, without a plausible underlying physiological mechanism (Post, 1994).

Finally, the door remains wide open to apply the rich arsenal of visual psychophysics to the study of mental illness more directly. In this regard, most of the fundamental questions remain unprobed. For instance, it is unclear whether there is a link between visual sensitivity and emotional sensitivity. Moreover, the functional significance of low GABA levels in depression is unclear. Are they epiphenomenal? Causal? Could training and in- tervention treatments on psychophysical tasks counteract some of the psychophysical effects of mental illness? In turn, could this perhaps alleviate the mental illness itself? As the answers to these essential questions are unknown, the Golomb et al. (2009) study might well become paradigmatic. Research of this kind could lead to a more integrated future in which the study of mental illness is closely intertwined with the study of basic visual processing, thereby providing a richer understanding of mental illness on a neural level.

\section{References}

Aaen-Stockdale CR, Thompson B, Huang PC, Hess RF (2009) Low-level mechanisms may contribute to paradoxical motion percepts. J Vis 9:9.1-9.14.

Anton-Erxleben K, Stephan VM, Treue S (2009) Attention reshapes center-surround receptive field structure in macaque cortical area MT. Cereb Cortex 19:2466-2478.

Betts LR, Taylor CP, Sekuler AB, Bennett PJ (2005) Aging reduces center-surround antagonism in visual motion processing. Neuron 45:361-366.

Bhagwagar Z, Wylezinska M, Jezzard P, Evans J, Ashworth F, Sule A, Matthews PM, Cowen PJ (2007) Reduction in occipital cortex gammaaminobutyric acid concentrations in medicationfree recovered unipolar depressed and bipolar subjects. Biol Psychiatry 61:806-812.

Born RT, Bradley DC (2005) Structure and function of visual area MT. Annu Rev Neurosci 28:157-189.

Golomb JD, McDavitt JR, Ruf BM, Chen JI, Saricicek A, Maloney KH, Hu J, Chun MM, Bhagwagar Z (2009) Enhanced visual motion perception in major depressive disorder. J Neurosci 29:90729077.

Kessler RC, Berglund P, Demler O, Jin R, Koretz D, Merikangas KR, Rush AJ, Walters EE, Wang PS (2003) The epidemiology of major depressive disorder. JAMA 289:3095-3105.

Post F (1994) Creativity and psychopathology. A study of 291 world-famous men. Br J Psychiatry 165:22-34.

Sanacora G, Mason GF, Rothman DL, Behar KL, Hyder F, Petroff OA, Berman RM, Charney DS, Krystal JH (1999) Reduced cortical gammaaminobutyric acid levels in depressed patients determined by proton magnetic resonance spectroscopy. Arch Gen Psychiatry 56:10431047.

Spencer J, O’Brien J, Riggs K, Braddick O, Atkinson J, Wattam-Bell J (2000) Motion processing in autism: evidence for a dorsal stream deficiency. Neuroreport 11:2765-2767.

Tadin D, Lappin JS, Gilroy LA, Blake R (2003) Perceptual consequences of centre-surround antagonism in visual motion processing. Nature 424:312-315.

Tadin D, Kim J, Doop ML, Gibson C, Lappin JS, Blake $\mathrm{R}$, Park S (2006) Weakened center-surround interactions in visual motion processing in schizophrenia. J Neurosci 26:11403-11412.

Thiele A, Distler C, Korbmacher H, Hoffmann KP (2004) Contribution of inhibitory mechanisms to direction selectivity and response normalization in macaque middle temporal area. Proc Natl Acad Sci U S A 101:9810-9815. 